\title{
Identification the appearance quality of rice kernels by vision technology and neural network classifier
}

\author{
Ali Dabaghi ${ }^{1}$, Mohamad Hadi Khoshtaghaza ${ }^{1}$ *, Mohamad Reza Alizadeh ${ }^{2}$, Hemad Zareiforoush ${ }^{3}$ \\ ${ }^{1}$ Biosystems Engineering Department, Tarbiat Modares University, Tehran, Iran \\ ${ }^{2}$ Department of Agricultural Engineering, Rice Research Institute of Iran (RRII), Guilan, Iran \\ ${ }^{3}$ Department of Agricultural Mechanization Engineering, University of Guilan, Guilan, Iran \\ *Corresponding author E-mail: khoshtag@ modares.ac.ir
}

\begin{abstract}
In this study, the appearance quality of Hashemi variety of rice grains was evaluated using image processing and artificial neural network (ANN) classifier. Non-touching kernel images of different classes in a Hashemi rice sample were acquired using a flatbed scanner. Then preprocessing, segmentation, feature extraction and effective feature selection process were done on each objects of image. To categorized grains, various structures of ANN consisting network with one and two hidden layer with different hidden nodes, different training and transfer functions were considered. Results of validation stage showed ANN with 13-18-18-5 topology and LM training and tansig transfer functions had highest mean of classification accuracy (97.33\%) and the lowest value of RMSE (0.08361). It's concluded that the suggested method uses low cost equipment to identify quality of rice with acceptable accuracy. Results of this research can be used for fast and accurate grading and developing an efficient rice sorting system.
\end{abstract}

Keywords: Artificial Neural Network; Classification; Image Processing; Quality; Rice.

\section{Introduction}

Rice is an important and mature food product in the world especially in Asia which provides energy and considered as the main food. Rice is a major crop in Iran, too. The most important regions for producing rice are in the northern provinces of IRAN [1]. Among different varieties of rice, Hashemi variety has good marketability due to having appropriate flavor, size and cooking quality, and most widely grown by farmers in these regions. There is direct relationship between economic value and grain quality. The rice quality includes appearance quality (related to its shape, size and color), milling quality (which define as the quality of product derived from paddy) and cooking quality (that identify by some ways like the needed time for cooking grains, the elongation ratio within cooking, the amount of solid particles that remain in the water of cooked grains and the percent of water absorption of each grains) [2], [3]. Head rice yield (HRY) is one of the appearance quality factors which is related to the number of sound and broken kernels existed in a sample [4]. Broken rice kernels has less marketability and price rather than sound kernels. Rice kernels with a length of less than three fourths of healthy kernels are called as broken kernels [5]. Percentage of chalky and discolored grain are another appearance quality factors. Generally, rice kernels are translucent. If a segment of the white rice is opaque (not translucent), it is identified as chalky [6]. Although appearance of chalky kernels fade during cooking process but presence of chalky kernels decreased the quality and grain salability [7]. Discoloration (excessive browning and blacking) in rice kernels occurs due to harvesting in high grain moisture content, harvesting during presence of dew, inappropriate storage, delay or improper drying, development of fungi and biochemical process [8], [9]. Presence of some discolored kernels in sample of white rice has adverse effects on its appearance quality which effects on its economic value.

Traditionally, rice quality is determined manually by well-trained human inspectors which are tedious, time-consuming and non-consistent. Various inspectors make own their decision about what they feel. As a result, they might have high percent of error. This has generated the need for new methods that are simple, rapid, accurate, and nondestructive [10]. In recent years, researchers have been able to invented automatic methods based on camera and computer which have the ability to analyze the characteristic of the different products [11]. This methods called as computer vision system. There are five main components which are related to computer vision system such as illumination, acquisition of image, board of image capture (digitizer or frame grabber), processing and analyses of image [12].

Some of researchers have represented the computer vision application on distinguishing and classifying of grain and its relevant quality factors in case of bulk form or single kernel. Paliwal et al., [13] extracted shape and size features like compactness, roundness, ferret diameter, elongation, major and minor axis length, perimeter and area for some cereal grain like rye, barley, oats, Canadian Western Amber Durum (CWAD) wheat and Hard Red Spring (HRS) wheat. Then the extracted features were used in order to train different structures of ANN. The reported accuracy of classification was up to $88 \%$ for tested cereal grains by ANN networks with four layer structure. Visen et al., [14] categorized some Canadian cereal grains by extracting morphological features. They represented categorized accuracies of $98.7 \%$ for barley, $99.3 \%$ for CWRS wheat, $96.7 \%$ for CWAD wheat, $98.4 \%$ for oats, and 96.9 for rye which were acquired by using expert 
probabilistic ANN. Golpour et al., [15] developed an algorithm based on color features and ANN for identifying and classifying five Iranian bulk paddy, brown and rice varieties. Images of bulk sample were obtained by appropriate scanner. Result represented that the ANN with two hidden layers and topologies of 36-6-5-5, 36-9-6-5 and 36-6-6-5 had mean classification accuracy of 98.8, 100, and 100\% for paddy, brown and white rice varieties, respectively.

Aulakh and Banga, [10] developed an algorithm for determining purity percent of non-touching brown rice samples. After segmentation of scanned brown rice images, they changed to binary images for performing other morphological operations. The mean area (in pixel) of broken grains was lower than sound grains. Thus, an area based threshold value was selected to discriminate between sound and broken grains. By counting all kernels (before threshold) and sound kernels (after threshold) in sample image, the percent of purity was determined. A same method of morphological operations was conducted by Prajapati and patel, [16] and Ajay et al., [17]. In another study, Dalen, [18] had studied on identification of the dimension, amount of sound grains, and rice dimension distribution using flatbed scanning (FBS). Also Zareiforoush et al., [19] specified the best classification methods for grading of white rice kernels based on their lengths and degree of milling. Images of four various categories of non-touching milled rice consisting low-milled broken kernels (LMB), low-milled sound kernels (LMS), high-milled broken kernels (HMB), and high-milled sound kernels (HMS) were obtained by using a CCD camera. Fifty seven features (including 5 features belong to size and shape, 4 features belong to texture and 48 features belong to color) were extracted for each rice kernel. Four various techniques of classification consisting support vector machine (SVM), decision tree (DT), artificial neural networks (ANN) and Bayesian Network (BN) were used to classify samples. Results showed that all of the classification techniques had acceptable accuracy. Among them, BN with Hill Climber search algorithm had the lowest accuracy of 96.89\% and ANN with 12-5-4 topology had the highest accuracy of $98.72 \%$ for classification of samples. Also the classification accuracy of DT with REP algorithm and SVM with Universal Pearson VII kernel function was 97.5 and $98.48 \%$, respectively.

Yadav and Jindal, [20] monitored milling quality of ten Tahi rice varieties using image analyses. By a CCD camera, they calculated three size and shape features such as projected area, perimeter and length for each individual kernel. Ten Thai rice varieties were selected. At first, all of the paddy samples were dehusked. Then, dehusked samples (brown rice) were purred into a laboratory milling device in order to produce white rice. The milling time was adjusted between 0.5 to $2.5 \mathrm{~min}$ with intervals of 0.5 min to produce white rice with different degree of milling. The morphological features of projected area, length and perimeter were calculated for each object. For milling performance evaluation, mean gray level distribution of bulk sample was obtained from images and correlated with results of commercial whiteness meters that operate based on white rice surface reflectance of light. Results show that projected area of each kernel was useful parameter for calculating the HRY. Also the results showed that the gray level depended to milling degree and varieties. It was changed from 70 to 190. In all varieties, with increasing degree of milling, the amount of mean gray level was increased. In another research, potential of chalkiness determination was evaluated by Yoshioka et al., [21]. The study was conducted based on chalkiness rate and chalkiness position on each kernels. A SVM classifier was used to categorize grains. Results show that SVM classifier could identify the chalkiness rate with mean accuracy of $85.5 \%$. Also, accuracy of $90.2 \%$ was obtained by SVM for calculating chalkiness position on each kernel. The overall results indicated that vision technology is powerful tools for chalkiness identification.

A sample of white rice (Hashemi variety) may contain various percentages of white rice, yellow rice, gray or black rice, chalky rice and broken rice which effects on its marketability, quality and price. Literature review showed that there is no comprehensive research for determination all of the major quality parameters of rice. Thus, our research goal was to create a vision based program to determine amount of head white rice (HWR), head yellow rice (HYR), head gray rice (HGR), head chalky rice (HCR) and broken rice (BR) that existed in a sample.

\section{Materials and methods}

The steps of our vision based program are shown in Fig. 1. After preparation of samples, images of rice kernels were taken. Then the taken images were pre-processed and features related to morphology, texture and color of each object were calculated. In the next step, proper features were selected. Selected features were applied as inputs to ANN classifier for separating various classes and finally the classifier performance was evaluated.

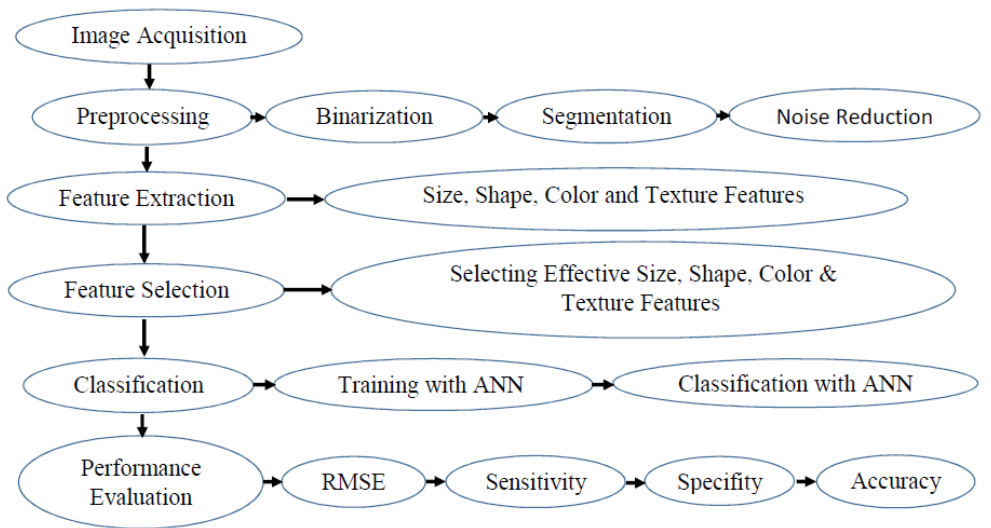

Fig. 1: Steps of Rice Image Processing Procedure.

\subsection{Sample preparation}

The amount of $4 \mathrm{~kg}$ sample of Hashemi variety containing five different classes including sound kernels (white, gray, yellow and chalky kernels) and broken kernels was prepared from the Rice Research Institute of Iran (RRII). The initial moisture content of the sample was between 11-14\% (w.b.) which was evaluated by a digital moisture meter (GMK model 303RS, Korea). The sample was then divided into four parts of $1 \mathrm{~kg}$ and each part was given to an expert in order to separate different classes in the sample. Finally, each separated class mixed together and 300 kernels from each class were randomly selected.

\subsection{Image acquisition}


For taken the images of rice kernels, a flatbed scanner (HP Scanjet 3570c, USA) with 300 dpi resolution and $2528 \times 3507$ pixels in JPG format was used. Each captured image of each class containing 300 kernels. The kernels were purred on the scanner glass and separated manually from each other. Then a black sheet was placed on them and their images were taken. Totally, images of 1500 kernels were obtained from all classes. Images of various classes of Hashemi rice are represented in Fig. 2, where Fig. 2-A, shows head white rice (HWR), Fig. 2-B, shows head chalky rice (HCR), Fig. 2-C, shows head gray rice (HGR), Fig. 2-D, shows head yellow rice (HYR) and Fig. 2-E, shows broken rice (BR). In order to display visual performance of suggested algorithm, an images containing combination of all classes in non-touching form were captured (see Fig. 2-F).

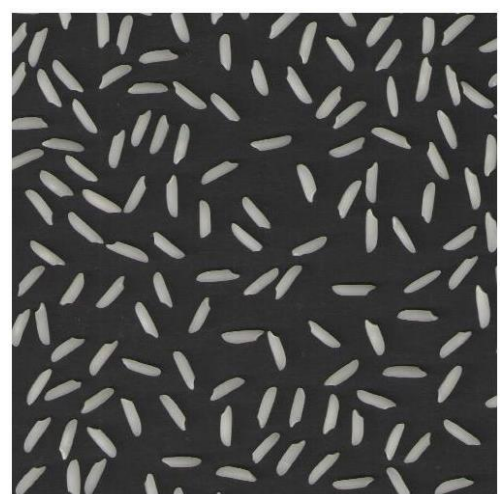

A) Head White Rice

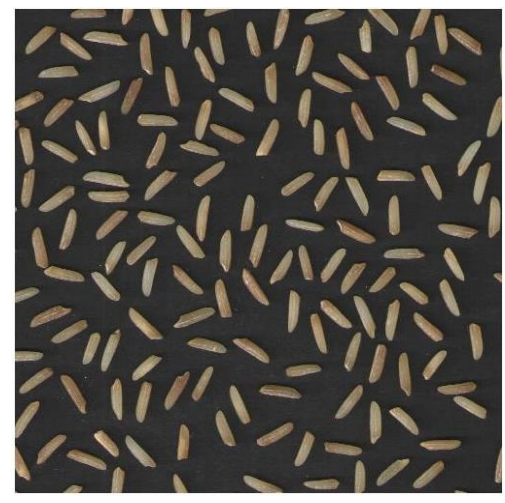

D) Head Yellow Rice

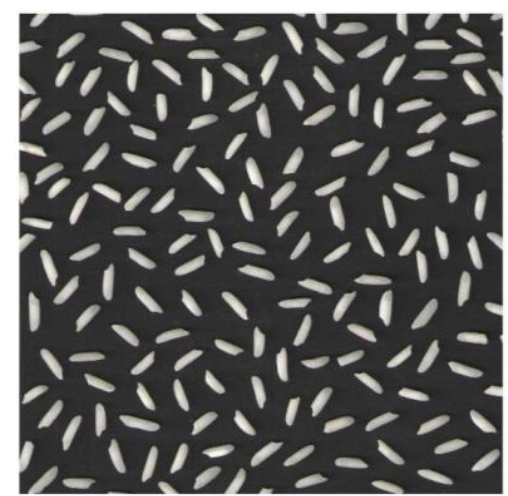

B) Head Chalky Rice

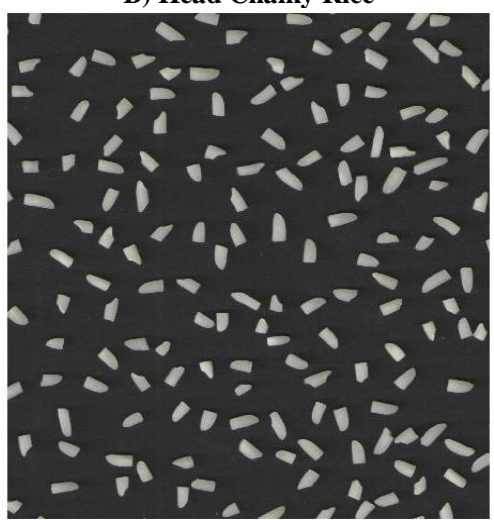

E) Broken Rice

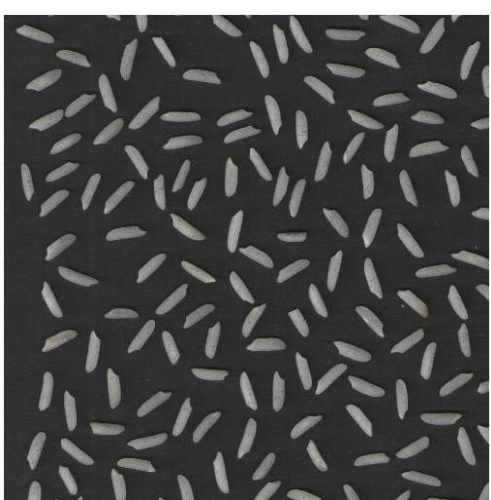

C) Head Gray Rice

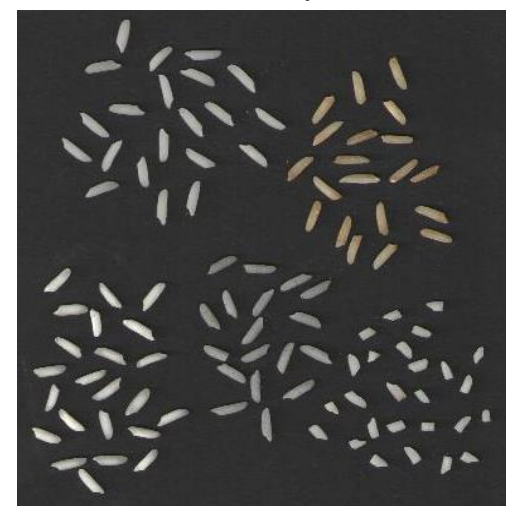

F) All Classes

Fig. 2: Images of Different Classes Which Existed in Hashemi Rice Sample.

\subsection{Pre-processing}

Preprocessing is a mathematical set of operations that is performed on a digital image to fit that image for a particular purpose (for example feature extraction). In this study, preprocessing procedure consist of binarization of RGB image, noise removal, filling inside of each object (kernel), segmentation and labeling of each kernel (head or broken) from other in the image. The RGB images binarization were done based on Otsu threshold method. In this method, at first, a threshold coefficient value was applied on gray level intensity of image. Then intensity of each pixel compared with selected threshold value. If pixel intensity was greater than threshold, the pixel intensity becomes 1 (white) and vice versa. In our research, the threshold coefficient value of 0.35 was selected. At the next stage, the generated noises caused by factors such as converting color images to binary images, the presence of small particles on the scanner glass and etc., were removed from the images. Then, in order to extract correct data related to object's morphology (size and shape), filled inside of each object. Finally, all objects (kernels) in the image were separately segmented and labeled to analyze each kernel. The above steps are shown in Fig. 3.

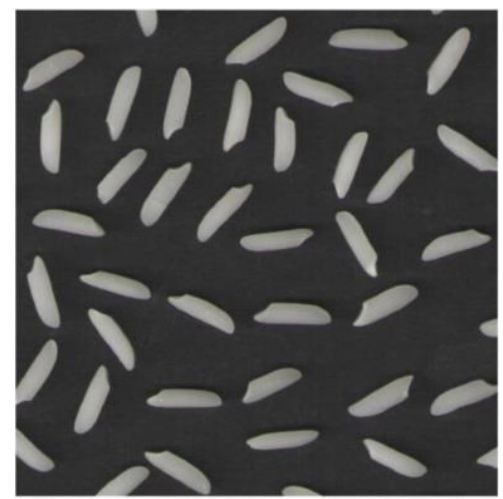

A) Original Image

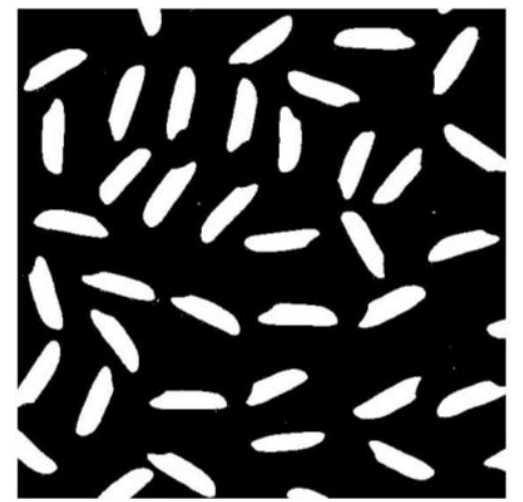

B) After Binarization

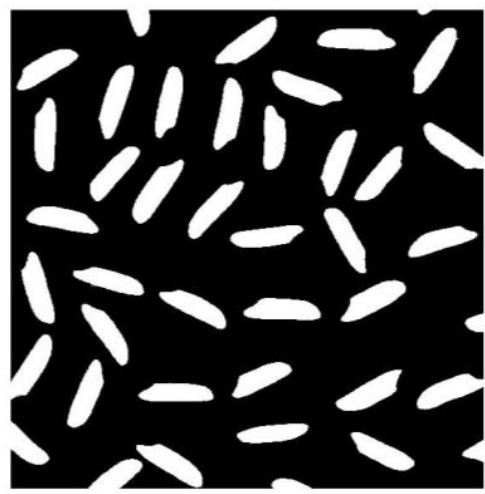

C) After Noise Reduction 


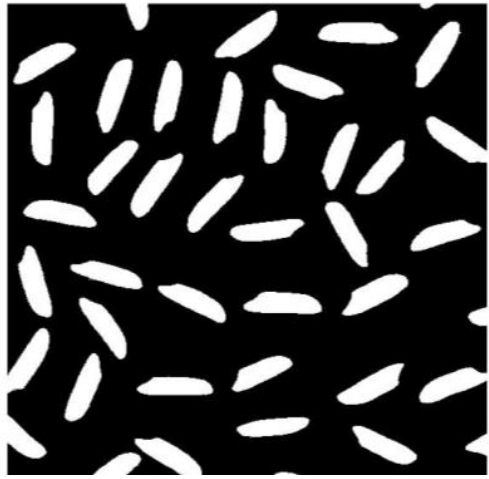

D) After Filling Holes

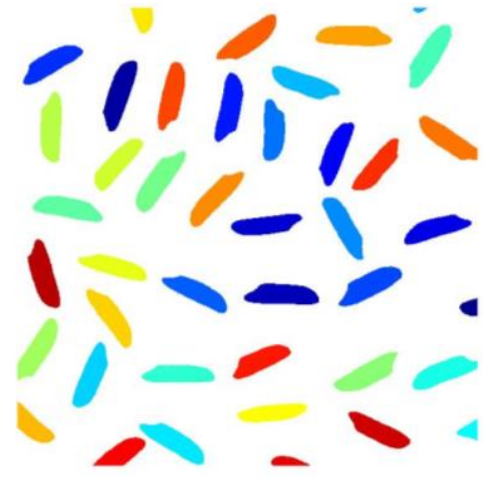

E) After Labeling

Fig. 3: Different Steps of Preprocessing of Hashemi Head White Rice.

\subsection{Feature extraction}

This step of image processing is done in order to describe, identify or classify and separation of the objects. In this paper, features related to size, shape were extracted from each object (see Table 1) [22], [23]. The RGB and HSI color spaces were used for color feature extraction and six statistical descriptor namely mean, variance, standard deviation, skewness, kurtosis and range were extracted from each related component (Table 2-A). Totally 36 color features (6 related component $\times 6$ statistical descriptor) were extracted from each object [24]. Texture analyze of each object was done by extracting gray level co-occurrence matrix (GLCM). For calculating the GLCM, the occurrence probability of pair of pixels with specific structure which arranged next to each other, must be calculated. This structure included a relative distance of $d$ (in pixel) and orientation of $\theta(\theta=0,45,90$ and 135) between pair of pixels that are in the neighborhood of each other. In this study, the distance of $d=1$ and orientation of $\theta=0$ was selected for calculating the GLCMs. Then, ten statistical features were extracted according to Table 2 -B. Thus, a total of 60 texture features ( 10 statistical features $\times 1$ orientations $\times 6$ relevant component $)$ were extracted from each object in each relevant component of R, G, B, H, S and I [24]. In sum, 110 features including 14 size and shape, 36 color feature and 60 texture feature were extracted for each object in the image.

Table 1: The Extracted Size and Shape Feature From Each Object [22], [23]

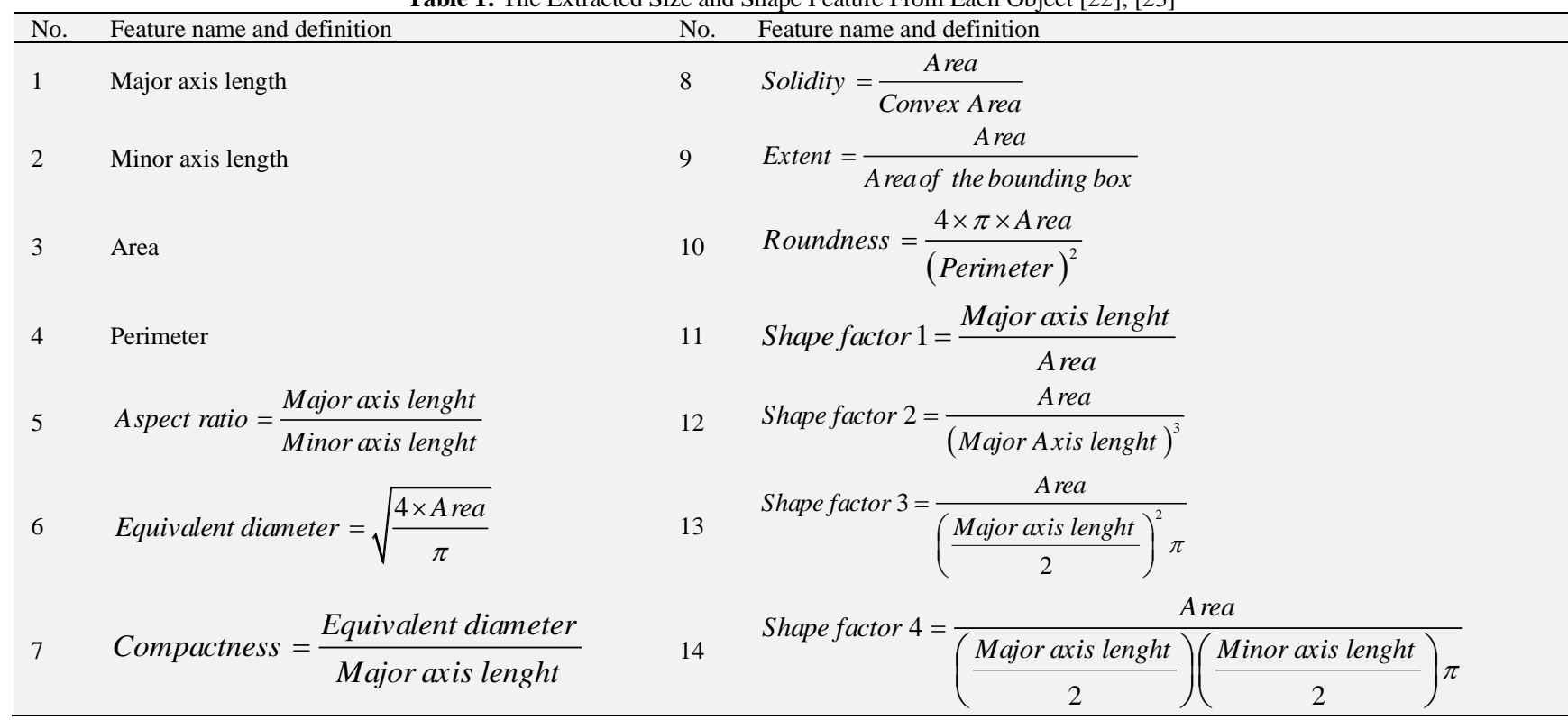

Table 2: Used Statistical Descriptor for Extracting Color and Texture Features [24]

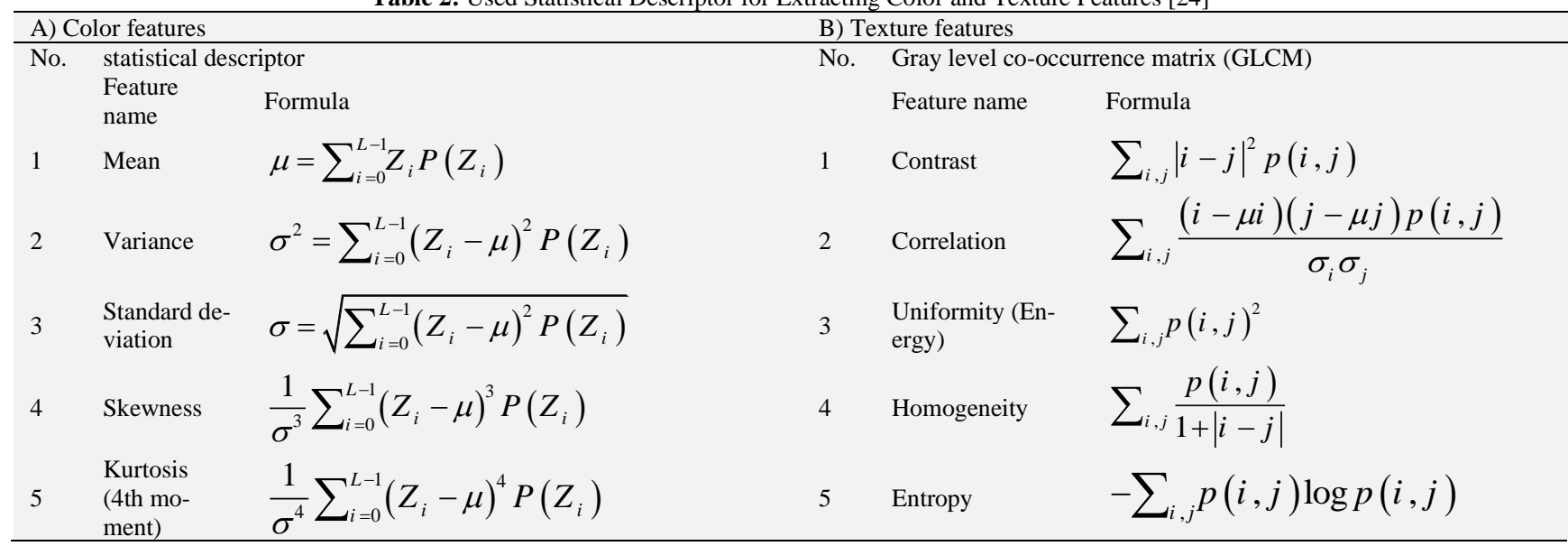




\begin{tabular}{|c|c|c|c|c|c|}
\hline \multirow[t]{5}{*}{6} & Range & $\max \left(L \times P\left(Z_{i}\right)\right)-\min \left(L \times P\left(Z_{i}\right)\right)$ & 6 & $\begin{array}{l}\text { Maximum of } \\
\text { probability }\end{array}$ & $\max (p(i, j))$ \\
\hline & & & 7 & Dissimilarity & $-j \mid p(i, j)$ \\
\hline & & & 8 & Cluster shade & $\sum_{i, j}((i-\mu i)+(j-\mu j))^{3} p(i, j)$ \\
\hline & & & 9 & $\begin{array}{l}\text { Cluster promi- } \\
\text { nence }\end{array}$ & $\sum_{i, j}((i-\mu i)+(j-\mu j))^{4} p(i, j)$ \\
\hline & & & 10 & Variance & $\sum_{i, j}(i-\mu i)^{2} p(i, j)$ \\
\hline
\end{tabular}

$L, \mu, \sigma, Z_{i}, P\left(Z_{i}\right)$ and $p(i, j)$ are dimension of image, mean of intensity, standard deviation, intensity levels, the number of possible intensity levels and GLCM matrix, respectively.

\subsection{Effective feature selection}

All of the 110 extracted features from each object are not appropriate for good classification and they may decrease accuracy and increase processing time of classification. The effective feature selection process is so important step and selected features must be considered as input of classifier. Several methods like wrapper methods, factor analysis (FA), sensitivity analysis (SA), correlation based feature selection (CFS) and principle component analysis (PCA) are usable for choosing best features [25 - 27]. Best features of our research was selected by CFS method. Generally, in feature selection methods, a feature evaluator and search method are needed. The feature evaluator specified which methods was used to dedicate a merit to each subset of features. When CFS method was used as evaluator, the following hypothesis was considered: "A good feature subset is one that contains features highly correlated with (predictive of) the class, yet uncorrelated with (not predictive of) each other" [28]. Also, the search method is an algorithm which determine searching style. In the current study best first search algorithm (BFS) was selected because of it allows backtracking along the search path. It was applied on the extracted shape, size, color and texture features of different classes of Hashemi variety using "CfsSubsetEval" evaluator in WEKA software [29]. The output results of CFS method indicated that the number of features decreased from 110 to 13 , including 5 for shape and size features, 4 for color features and 4 for texture features. The selected features name are shown in Table 3.

Table 3: Superior Features Which Selected by CFS Algorithm

\begin{tabular}{lll}
\hline Size \& shape features & Color features & Texture features \\
\hline Area & $\mathrm{S}_{\mathrm{hsi}}$ Mean & $\mathrm{B}_{\mathrm{rgb}}$ Correlation \\
Shape factor 2 & $\mathrm{B}_{\mathrm{rgb}} \mathrm{Std}$ & $\mathrm{H}_{\mathrm{hsi}}$ Maximum of probability \\
Equivalent diameter & $\mathrm{S}_{\mathrm{hsi}}$ Std & $\mathrm{S}_{\mathrm{his}}$ Energy \\
Major axis lenght & $\mathrm{B}_{\mathrm{rgb}}$ Variance & $\mathrm{S}_{\mathrm{hsi}}$ Entropy \\
Perimeter & & \\
\hline
\end{tabular}

* S and B are related to the HSI and RGB color components, respectively.

\subsection{Classification}

Many techniques like Decision Tree (DT), K-Nearest Neighbor (KNN), Fuzzy Logic (FL), Artificial Neural Network (ANN) and Support Vector Machine (SVM) are used by researchers for classification [26], [30]. An ANN is an idea for data processing which has been improved by biological neural system and processed data like human brain [31]. One of the most advanced neural model is multi-layer perceptron (MLP) which can simulate transitional function of human brain and were applied in various application like prediction and classification [27]. Each simple neural network consist of input and output layer which is used to solve simple problems, while in MLP, there are additional layers (or layer) called hidden layers (or layer) which helps to solve complex problems. MLP networks belong to feed forwards network. This network is trained based on back propagation algorithm. Also different back-propagation algorithm such as SCG (scaled conjugate gradient), LM (Levenberg-Marquardt), GDM (gradient descent with a momentum), GDX (gradient descent with momentum and adaptive learning rate back-propagation), OSS (one step secant) and etc. are available. They are used for minimizing the error [27], [31]. For classification of our rice sample, MLP network was used.

The numbers of neurons were 13 in input layer which were same to the number of effective chosen features as represented in Table 3 . The number of neurons in output layer was five, which was equal to the number of different existed classes, including head white rice (HWR), head chalky rice (HCR), head gray rice (HGR), head yellow rice (HYR) and broken rice (BR), in Hashemi rice sample. Generally, the number of hidden layer and hidden nodes are directly affected on accuracy of classification. Hence, determining these optimal numbers are crucial steps in designing classifier and usually founded by trial and error methods [15]. As well as, various transfer functions like purelin, logsig and tansig permit the network to know linear and nonlinear relationships between input and output data. Consequently they may have effect on performance of ANN and must be considered [32].

\subsection{Performance evaluation}

In this study, about $75 \%$ of the samples ( 225 kernels for each class) were randomly selected for training the network, while the rest of the samples were used as test set. Different structures includes four training algorithm (GDX, LM, OSS and SCG), one and two hidden layer with different hidden node (2- $4-6-\ldots-18$ and 2, 2- 4, $4-5,5-\ldots-18,18)$ and three transfer function (purelin, logsig and tansig) were tested in order to determine the optimal performance for the network. The best network topology was chose based on overall accuracy and root mean squared error (RMSE) indexes [26]. All networks were run three times and the mean of overall accuracy and RMSE were considered as decision indexes. Also, to describe performance of the best selected network, three statistical indicators such as accuracy $\left(A_{c}\right)$, sensitivity $\left(S_{e}\right)$, specificity $\left(S_{p}\right)$ and confusion matrix were used. Sensitivity is related to positive objects which correctly predicted, and specificity is related to negative objects which correctly predicted. Whereas accuracy is related to both positive and negative objects which correctly predicted. Their mathematical relation are shown by Equations (1) to (4) [19], [31]

$A_{c}=\frac{T P+T N}{T P+F P+T N+F N} \times 100$. 
$S_{e}=\frac{T P}{T P+F N} \times 100$

$S_{P}=\frac{T N}{F P+T N} \times 100$

$R M S E=\sqrt{\frac{1}{N} \sum_{i=1}^{N}\left(A_{i}-P_{i}\right)^{2}}$

Where, TP is true positive (the number of kernels whose their real class was positive and correctly classified as positive), TN is true negative (the number of kernels whose their real class was negative and correctly classified as negative by classifier), FP is false positive (the number of kernels whose their real class was negative and incorrectly classified as positive) and FN is false negative (the number of kernels whose their real class was positive and incorrectly classified as negative). Also $\mathrm{N}$ is total test set, $A_{i}$ is actual value and $P_{i}$ is predicted value.

\section{Results and discussion}

Results of accuracy mean and RMSE indexes obtained from different neural networks test with one hidden layer, different number of neurons, different training and transfer functions are represented in Fig. 4 and 5. According to Fig. 4, after network training and simulation of the tested data, the findings represented that structures with "purelin" and "tansig" transfer function had a passable accuracy range. Among them, the highest accuracy indexes were related to LM training function with "tansig" transfer functions, 10 and 16 number of neurons (13-10-5 \& 13-16-5 topologies). These values were 96.33 and 96.67 , respectively. In order to choose the best topology of one layer network, the RMSE of each topology were intended and network with lowest RMSE was selected. The value of RMSE index for 1316-5 topology was 0.1004 which was less than the value of RMSE for 13-10-5 topology with amount of 0.11176. So neural network with 13-16-5 topology (one layer with 16 neurons), LM training function and tansig transfer functions was selected as the best structure.

The results of accuracy mean and RMSE index for ANN with two hidden layer and different number of neurons, training and transfer functions are shown in Fig. 6 and 7. The worst performance were related to networks with logsig transfer functions which had minimum accuracy and maximum RMSE in all neurons and training functions. Like the findings acquired from one layer ANN, structures with "purelin" and "tansig" transfer function had an acceptable accuracy range. As represented in Fig. 6, topologies with 18 and 20 neurons in each hidden layer (13-18-18-5 \& 13-20-20-5) with tansig transfer function an LM training function had the greatest accuracy index with the values of 97.33 and 96.67, respectively. According to Fig. 7, the lowest value of RMSE was related to 13-18-18-5 topology with the amount of 0.08361 . So the best two hidden layer topology was 13-18-18-5 with Lm training function and tansig transfer function.

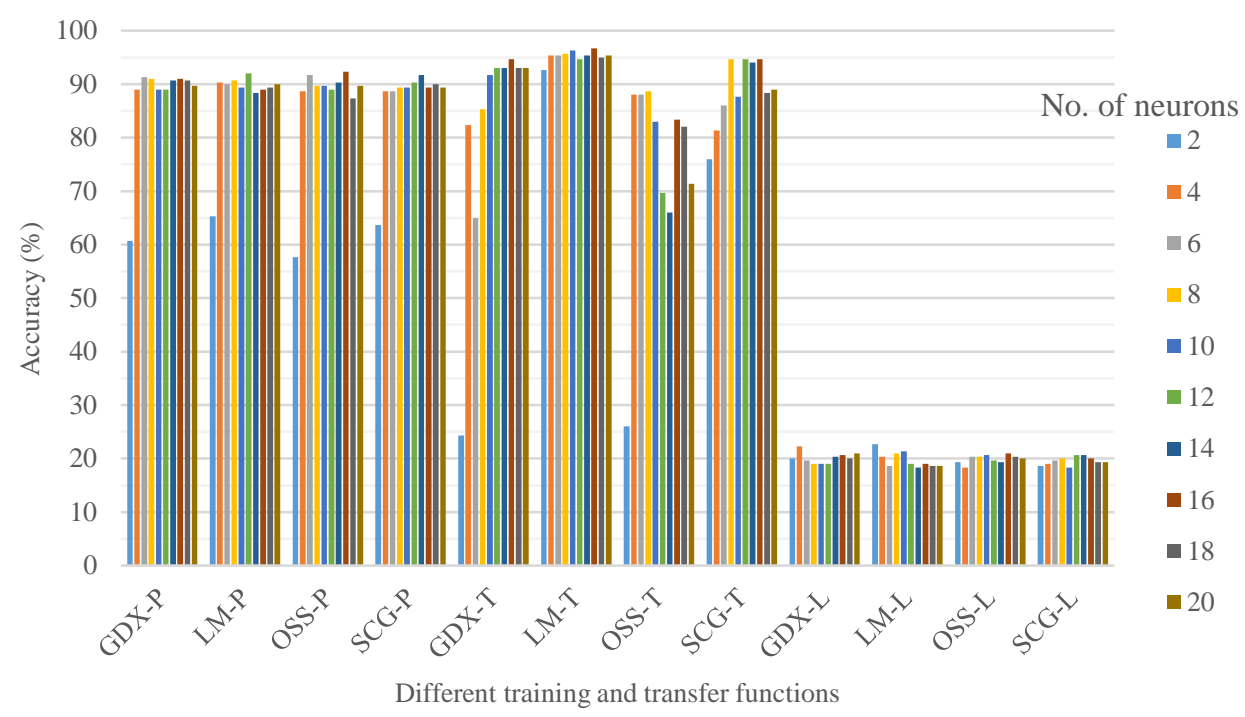

Fig. 4: Variation of Accuracy Index for ANN with One Hidden Layer and Combination of Different Number of Neurons, Training and Transfer Functions. (P, T and L Represents Purelin, Tansig and Logsig, Respectively 


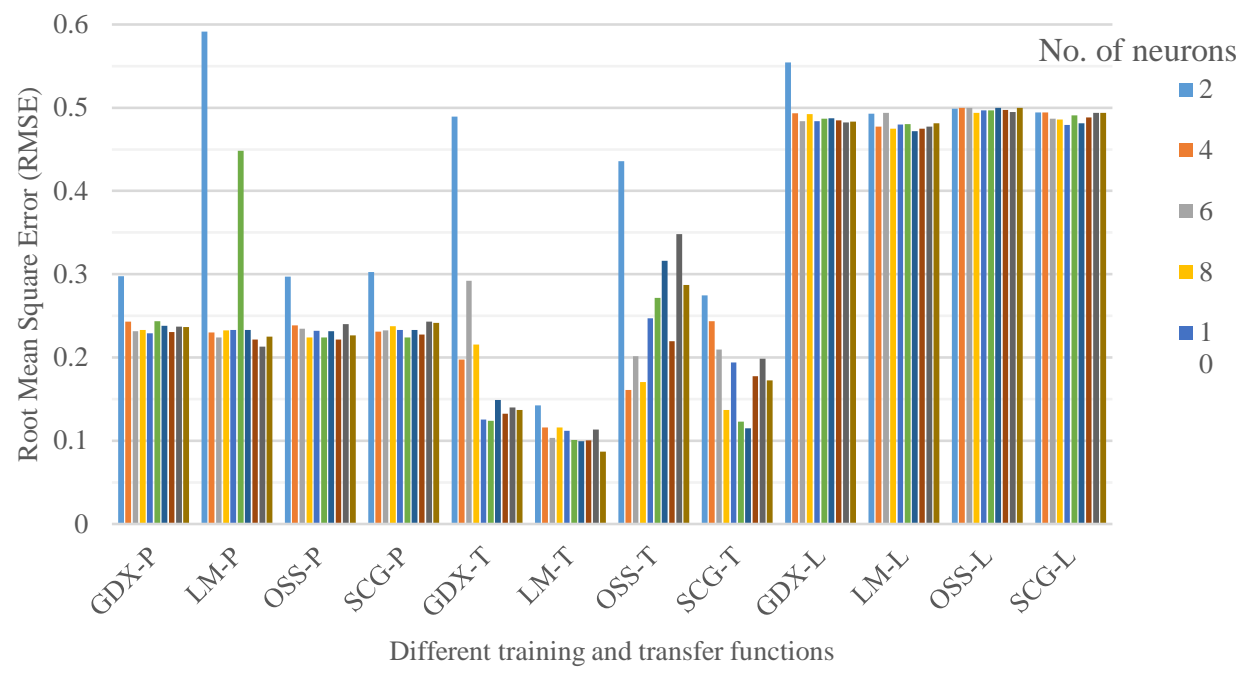

Fig. 5: Variation of the RMSE Index for ANN with One Hidden Layer and Combination of Different Number of Neurons, Training and Transfer Functions. (P, T and L Represents Purelin, Tansig and Logsig, Respectively).

Comparison between the best selected networks with one and two hidden layer showed that network with two hidden layer and 13-18-185 topology had maximum accuracy and minimum RMSE. Such network was appropriate for separating different classes which existed in the samples. Similar result was reported by Golpour et al., [15]. They used one layer neural network with 36-24-5, 36-7-7 and 36-5-5 topologies, Lm training function and logsig-purelin transfer functions for input and output layer in order to recognize and categorize of bulk paddy, brown rice and white rice of some Iranian varieties and reported mean classification accuracy of $93.3,98.9$ and $100 \%$, respectively. For improvement the classification accuracy, they examined network with two hidden layer and explained the mean accuracy up to 98.8\%. Liu et al., [33] recognized six paddy varieties based on ANN data mining method. They used ANN with tansig-logsig transfer function for classification and reported average classification accuracy of $84.43 \%$. Similar studies were done by Silva and Sonnadara, [34]; Shantaiya and Ansari, [35]; Rexce and Usha Kingsly Devi, [30] and Prajapati and Patel, [16] for identification of various rice types by ANN. In another research, Zareiforoush et al., [19] specified the best classification methods for grading of white rice grain based on length and degree of milling of the white rice kernels. The 12-5-4 topology of ANN was selected as superior topology with the mean accuracy of $98.72 \%$ which was suitable for classification of different quality grades of milled rice including (low-milled sound grain (LMS), lowmilled broken grain (LMB), high-milled sound grain (HMS) and high-milled broken grain (HMB)).

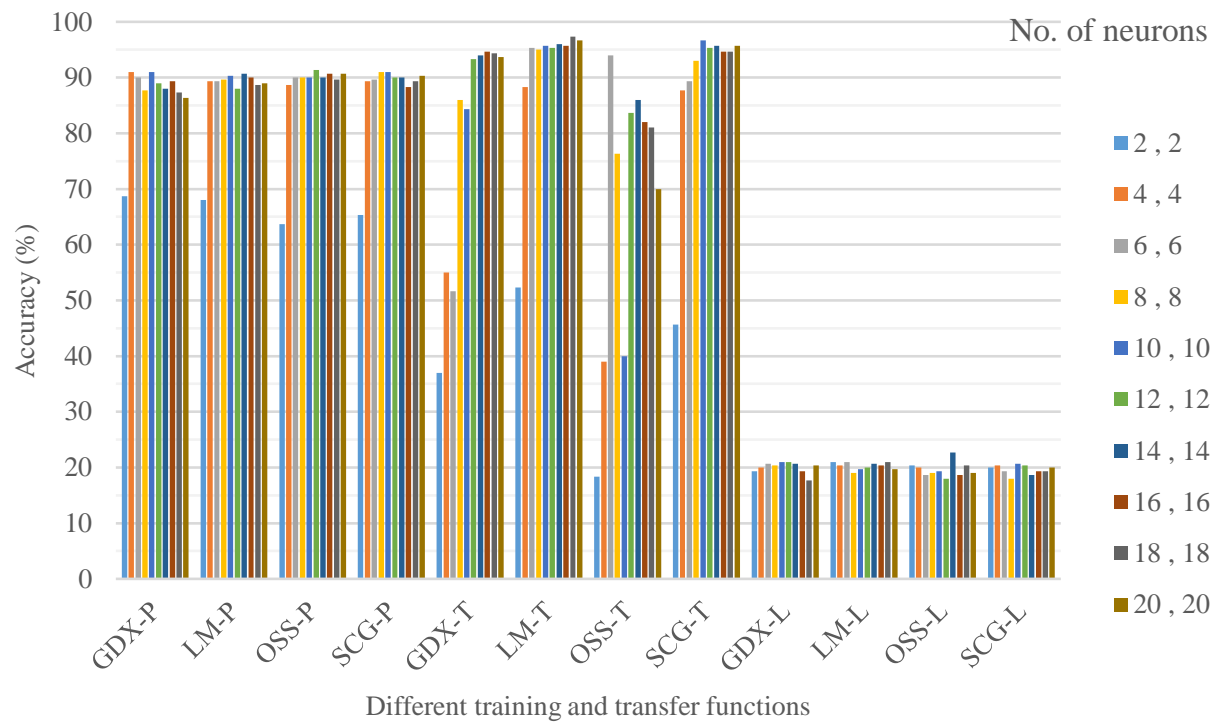

Fig. 6: Variation of the Accuracy Index for ANN with Two Hidden Layer and Combination of Different Number of Neurons, Training and Transfer Functions. (P, T and L Represents Purelin, Tansig and Logsig, Respectively). 


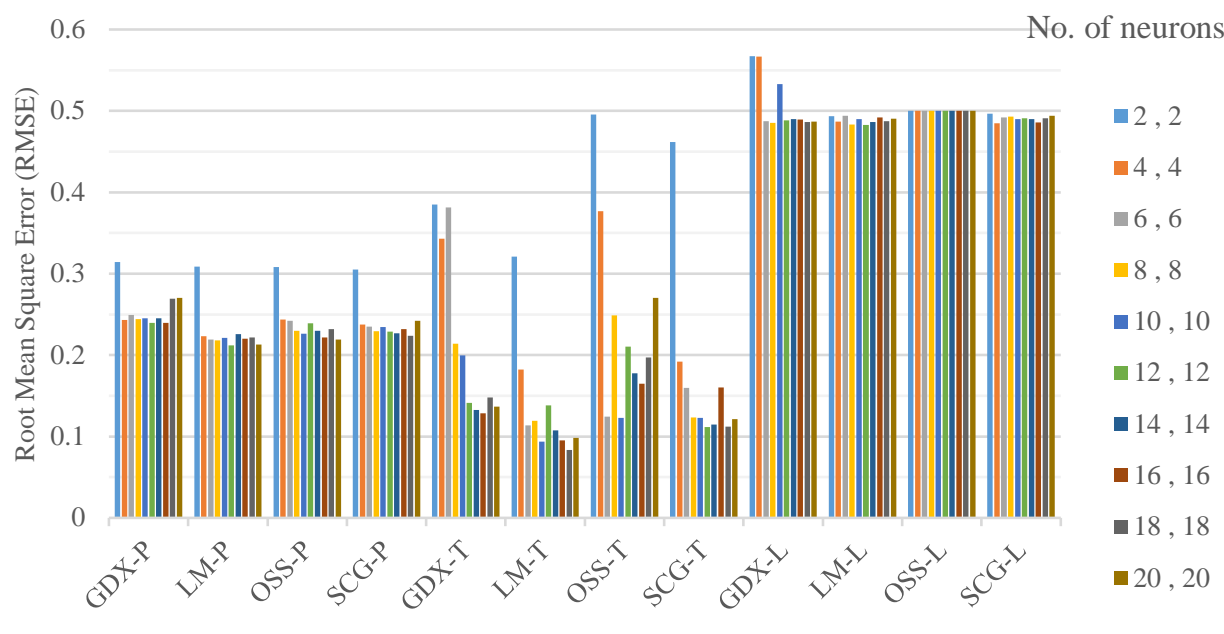

Fig. 7: Variation of the RMSE Index for ANN with Two Hidden Layer and Combination of Different Number of Neurons, Training and Transfer Functions. (P, T and L Represents Purelin, Tansig and Logsig, Respectively).

The chosen topology was examined several times and the confusion matrix values and three statistical parameters (like accuracy, specificity and sensitivity) associated to the best performance were reported in Tables 4 and 5. Due to obtained results from tables 4 and 5, the classifier sensitivity index $\left(\mathrm{S}_{\mathrm{e}}\right)$ for HWR, HCR, HGR, HYR and BR classes were 97.33, 100, 96, 97.22 and $95.12 \%$, respectively. The classifier specificity index $\left(\mathrm{S}_{\mathrm{p}}\right)$ for HWR, HCR, HGR, HYR and BR classes was equal to 98, 99.34, 99, 100 and $100 \%$, respectively. Also, the classifier accuracy index in grading of Hashemi rice grain into HWR, HCR, HGR, HYR and BR classes was 92.4, 97.26, 96, 100 and $100 \%$, respectively. The lowest accuracy percent were related to HWR and HGR classes which showed that the ability of created algorithm in classification of these classes was lower that other classes. Also the results indicated that the highest classification accuracy were related to HYR and BR classes using suggested classification algorithm. In order to display visual performance of suggested algorithm, an image includes all classes was captured and given as input to the algorithm. Finally, the algorithm with the selected topology was run and findings of visual classification of image are represented in Fig. 8. As shown in Fig. 8 and Table 5, the offered algorithm could be perform as a great deal of separation of classes from each other. The overall results showed that created structure was able to classify rice samples with acceptable accuracy and it can be used as monitoring tool to evaluate rice kernels.

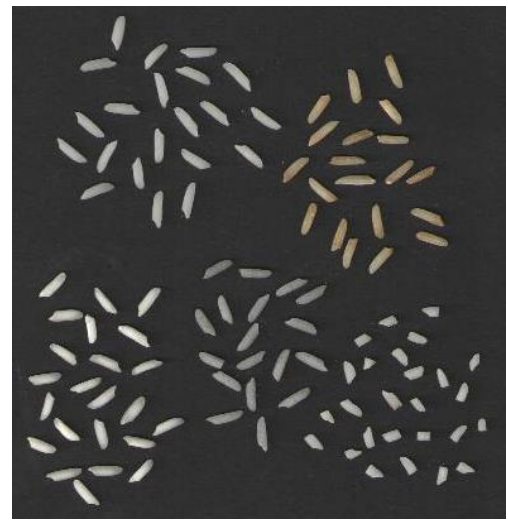

A) All Classes

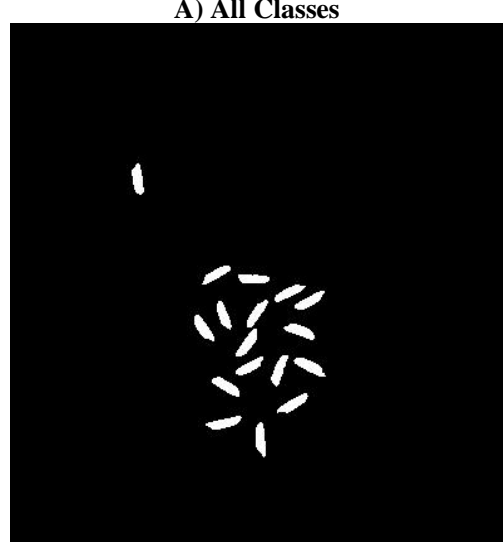

D) Head Gray Rice

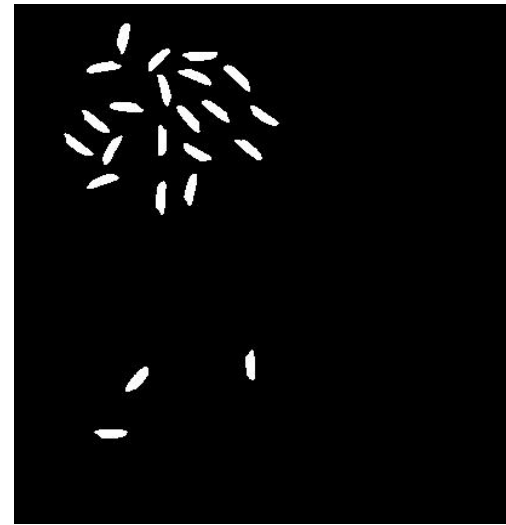

B) Head White Rice

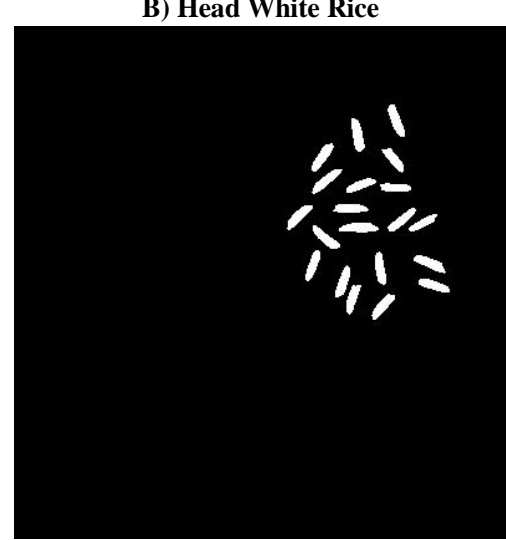

E) Head Yellow Rice

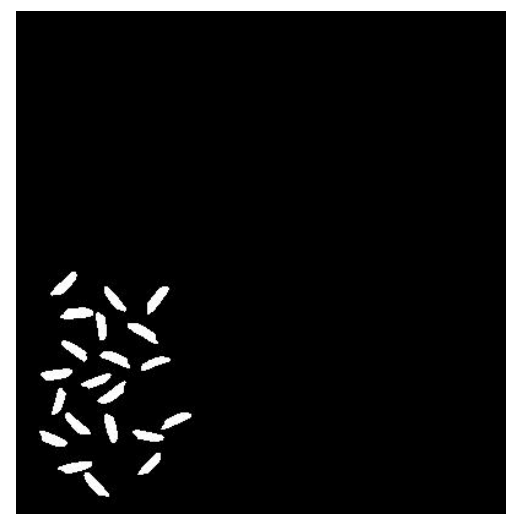

C) Head Chalky Rice

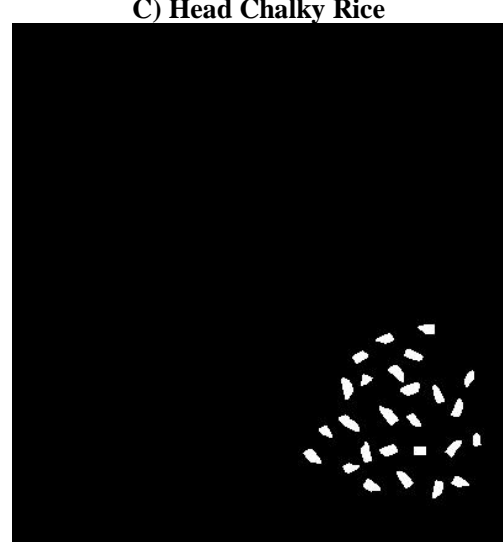

F) Broken Rice

Fig. 8: A) Image of All Classes, B) to F) Represent Results of Algorithm Classification. 
Table 4: The Confusion Matrix of 13-18-18-5 ANN Topology

\begin{tabular}{|c|c|c|c|c|c|}
\hline Predicted classes / Actual classes & HWR & HCR & HGR & HYR & $\mathrm{BR}$ \\
\hline HWR & 73 & 0 & 3 & 0 & 3 \\
\hline HCR & 1 & 71 & 0 & 0 & 1 \\
\hline HGR & 1 & 0 & 72 & 2 & 0 \\
\hline HYR & 0 & 0 & 0 & 70 & 0 \\
\hline $\mathrm{BR}$ & 0 & 0 & 0 & 0 & 78 \\
\hline
\end{tabular}

Table 5: Statistical Parameters Obtained from the Evaluation of 13-18-18-5 ANN Topology

\begin{tabular}{|c|c|c|c|}
\hline classes / statistical parameters & $S_{e(\%)}$ & $S_{p(\%)}$ & $A_{0}(\%)$ \\
\hline HWR & 97.33 & 98 & 92.40 \\
\hline HCR & 100 & 99.34 & 97.26 \\
\hline HGR & 96 & 99 & 96 \\
\hline HYR & 97.22 & 100 & 100 \\
\hline $\mathrm{BR}$ & 95.12 & 100 & 100 \\
\hline
\end{tabular}

\section{Conclusion}

In the present study, a technique was suggested for identification and classification of various existed classes in Hashemi rice samples (appearance quality of rice sample) based on image processing and ANN data mining method. To select the best ANN structure, different structure of ANN (including one and two hidden layer, different hidden node, different training and transfer function) were used. Comparison of accuracy and RMSE indexes showed network with the 13-18-18-5 topology, LM training function and tansig transfer functions was the best classifier, and their values were $97.33 \%$ and 0.08361 , respectively. Results of confusion matrix obtained from the best topology showed this techniques was very successful in detection of different classes existed in Hashemi rice sample. The suggested method with low equipment can be solved the problem of rapid and accurate determining rice quality. For future study, the finding of this research should be developed for other rice varieties and consequently it leads to design and development of rice inspection machine.

\section{Acknowledgement}

Authors are grateful to Tarbiat Modares University for funding and providing facilities related to image processing. Also we are grateful to Rice Research Institute of Iran (RRII) for providing the experimental samples for this study.

\section{References}

[1] M.R Alizadeh, S. Minaei, T. Tavakoli, M.H. Khoshtaghaza, Effect of de-awning on physical properties of paddy, Pakistan Journal of Biological Sciences 9 (2006), 1726-1731. https://doi.org/10.3923/pibs.2006.1726.1731.

[2] D. Mohapatra, S. Bal, Effect of degree of milling on specific energy consumption, optical measurements and cooking quality of rice, Journal of Food Engineering 80 (1) (2007) 119-125. https://doi.org/10.1016/j.jfoodeng.2006.04.055.

[3] M. Frei, P. Siddhuraju, K. Becker, Studies on the in vitro starch digestibility and glycemic index of six different indigenous rice cultivars from the Philippines, Food Chemistry 83 (3) (2003) 395-402. https://doi.org/10.1016/S0308-8146(03)00101-8.

[4] M.R. Alizadeh, A. Dabbaghi, F. Rahimi Ajdadi, Effect of final paddy moisture content on breaking force and milling properties of rice varieties, Elixir Agriculture 36 (2011) 3186-3189.

[5] H. Zareiforoush, M. Komarizadeh, M.R. Alizadeh, Effects of crop-machine variables on paddy grain damage during handling with an inclined screw auger, Biosystems Engineering 106 (3) (2010) 234-242. https://doi.org/10.1016/j.biosystemseng.2010.02.008.

[6] T. Tashiro, I.F. Wardlaw, The effect of high temperature on kernel dimensions and the type and occurrence of kernel damage in rice, Australian Journal of Agricultural Research, 42 (1991), 485-496. https://doi.org/10.1071/AR9910485.

[7] K. Bhavesh, Analysis of rice grains through digital image processing, International Journal of Scientific Research in Science and Technology 1 (1) (2015) 1-3.

[8] C.M. Christensen, C.M. Kaufmann, Deterioration of stored grains by fungi, Annual Review of Phytopathology 3 (1965) 69-84. https://doi.org/10.1146/annurev.py.03.090165.000441.

[9] N.M. Sahay, S. Gangopadhyay, Effect of wet harvesting on biodeterioration of rice, Cereal Chemistry 62 (2) (1985) 80-83.

[10] J.S. Aulakh, V. Banga, Percentage purity of rice sample by image processing, International conference on trends in electrical, electronics and power engineering (ICTEEP), July 15-16, Singapore (2012) 15-16.

[11] H.H. Wang, D.W. Sun, Evaluation of the functional properties of cheddar cheese using a computer vision method, Journal of Food Engineering 49 (1) (2001) 47-51. https://doi.org/10.1016/S0260-8774(00)00183-7

[12] H.H. Wang, D.W. Sun, Correlation between cheese meltability determined with a computer vision method and with Arnott and Schreiber tests, Journal of Food Science 67 (2) (2002) 745-749. https://doi.org/10.1111/j.1365-2621.2002.tb10670.x.

[13] J Paliwal, N.S. Visen, D.S. Jayas, AE-Automation and emerging technologies: Evaluation of neural network architectures for cereal grain classification using morphological features, Journal of Agricultural Engineering Research 79 (4) (2011) 361-370. https://doi.org/10.1006/jaer.2001.0724

[14] N.S. Visen, J. Paliwal, D.S. Jayas, N.D.G. white, Specialist neural networks for cereal grain classification, Biosystems Engineering 82 (2002) $151-$ 159. https://doi.org/10.1006/bioe.2002.0064.

[15] I. Golpour, R. Chayjan, Identification and classification of bulk paddy, brown, and white rice cultivars with colour features extraction using image analysis and neural network, Czech Journal of Food Sciences 32 (3) (2014) 280-287. https://doi.org/10.17221/238/2013-CJFS.

[16] B.B. Prajapati, S. Patel, Algorithmic approach to quality analysis of Indian basmati rice using digital image processing, International journal of emerging technology and advanced engineering 3 (3) (2013) 503-504.

[17] G. Ajay, M. Suneel, K.K. Kumar, P.S. Prasad, Quality evaluation of rice grains using morphological methods, International journal of soft computing and engineering 2 (2013) 35-37.

[18] V.G. Dalen, Determination of the size distribution and percentage of broken kernels of rice using flatbed scanning and image analysis, Food Research International 37 (1) (2004) 51-58. https://doi.org/10.1016/j.foodres.2003.09.001.

[19] H. Zareiforoush, S. Minaei, M.R. Alizadeh, A. Banakar, Qualitative classification of milled rice grains using computer vision and metaheuristic techniques, Journal of Food Science and Technology 53 (1) (2016) 118-131. https://doi.org/10.1007/s13197-015-1947-4.

[20] B.K. Yadav, V. Jindal, Monitoring milling quality of rice by image analysis, Computers and Electronics in Agriculture 33 (1) (2001) 19-33. https://doi.org/10.1016/S0168-1699(01)00169-7.

[21] Y. Yoshioka, H. Iwata, M. Tabata, S. Ninomiya, R. Ohsawa, Chalkiness in rice: potential for evaluation with image analysis, Crop Science 47 (2007) 2113-2120. https://doi.org/10.2135/cropsci2006.10.0631sc. 
[22] J.D. Guzman, E.K. Peralta, Classification of Philippine rice grains using machine vision and artificial neural networks, World conference on agricultural information and IT, Tokyo, Japan, (2008) 41-48.

[23] A. Pazoki, Z. Pazoki, Classification system for rain fed wheat grain cultivars using artificial neural network, African Journal of Biotechnology 10 (41) (2011) 8031-8038. https://doi.org/10.5897/AJB11.488.

[24] K. Mollazade, M. Omid, F. Akhlaghian Tab, Y. Rezaei Kalaj, S. Mohtasebi, M. Zude, Analysis of texture-based features for predicting mechanical properties of horticultural products by laser light backscattering imaging, Computers and Electronics in Agriculture 98 (2013) 34-45. https://doi.org/10.1016/j.compag.2013.07.011.

[25] A. Fielding, Cluster and classification techniques for the biosciences, Cambridge University Press, UK, 2007.

[26] K. Mollazade, M. Omid, A. Arefi, Comparing data mining classifiers for grading raisins based on visual features, Computers and Electronics in Agriculture 84 (2012) 124-131. https://doi.org/10.1016/j.compag.2012.03.004.

[27] M. Omid, A. Mahmoudi, M.H. Omid, Development of pistachio sorting system using principal component analysis (PCA) assisted artificial neural network (ANN) of impact acoustics, Expert Systems with Applications 37 (10) (2010) 7205-7212. https://doi.org/10.1016/j.eswa.2010.04.008.

[28] M. Hall, Correlation-based feature selection for machine learning, PhD Thesis, University of Waikato, Hamilton, New Zealand, 1999.

[29] M. Hall, E. Frank, G. Holmes, B. Pfahringer, P. Reutemann, I.H. Witten, The WEKA data mining software: an update, SIGKDD Explorations11 (1) (2009) 10-18. https://doi.org/10.1145/1656274.1656278.

[30] J. Rexce, K. Usha Kingsly Devi, Classification of milled rice using image processing, International Journal of Scientific and Engineering Research 8 (2) (2017) 10-15.

[31] N. Teimouri, M. Omid, K. Mollazade, A. Rajabipour, A novel artificial neural networks assisted segmentation algorithm for discriminating almond nut and shell from background and shadow, Computers and Electronics in Agriculture 105 (2014) 34-43. https://doi.org/10.1016/j.compag.2014.04.008.

[32] A. Khoshroo, A. Arefi, A. Masoumiasl, GH.H. Jowkar, Classification of wheat cultivars using image processing and artificial neural networks, Agricultural Communications 2 (1) (2014) 17-22.

[33] Z. Liu, F. Cheng, Y. Ying, X. Rao, Identification of rice seed varieties using neural network, Journal of Zhejiang University Science B. 6, (11) (2005) 1095-1100. https://doi.org/10.1631/jzus.2005.B1095.

[34] C.S. Silva, U. Sonnadara, Classification of rice grains using neural networks, Proceedings of Technical Sessions 29 (2013) 9-14.

[35] S. Shantaiya, U. Ansari, Identification of food grains and its quality using pattern classification, 12th IEEE International Conference on Communication Technology (ICCT), Nanjing, China, 11-14 November 2010. 